\title{
Effects of symmetry crossover in quantum transport in graphene and nanotube
}

\author{
By Tsuneya Ando \\ Department of Physics, Tokyo Institute of Technology, \\ 2-12-1 Ookayama, Meguro-ku, Tokyo 152-8551, Japan
}

A brief review is given on transport properties of carbon nanotubes mainly from a theoretical point of view. The topics include an effective-mass description of electronic states, and the absence of backward scattering and the presence of a perfectly conducting channel except for scatterers with a potential range smaller than the lattice constant. The dynamical conductivity in the presence of a perfect channel and effects of symmetry crossover are also discussed.

Keywords: graphite, carbon nanotube, impurity scattering, Berry phase

\section{Introduction}

Transport properties of carbon nanotubes are quite interesting. In metallic nanotubes there is no backscattering even in the presence of scatterers unless their potential range is smaller than the lattice constant (Ando \& Nakanishi 1998). When there are several bands at the Fermi level, interband scattering appears, but a perfectly conducting channel transmitting through the system without being scattered back exists and the conductance is always larger than $2 e^{2} / \pi \hbar$ (Ando \& Suzuura 2002). The purpose of this paper is to give a brief review of recent theoretical study on such unique transport properties.

In $\S 2$, an effective-mass description of electronic states of a monolayer graphite sheet or graphene and carbon nanotubes is briefly reviewed. In $\S 3$, the absence of backward scattering and the presence of a perfectly conducting channel, making a metallic nanotube a perfect and ballistic conductor even in the presence of scatterers, are discussed. In $\S 4$, singular behaviors of the dynamical conductivity are discussed. In $\S 5$, effects of symmetry-breaking perturbations and associated symmetry crossover are discussed.

\section{Neutrino description}

A monolayer graphite sheet has a honeycomb lattice with two atoms (denoted by $A$ and $B$ ) in a unit cell and is a zero-gap semiconductor in the sense that the conduction and valence bands consisting of $\pi$ states cross at $K$ and $K$ ' points of the Brillouin zone (Wallace 1947). The electronic states near the K point are described by the $\mathbf{k} \cdot \mathbf{p}$ equation $\gamma(\vec{\sigma} \cdot \hat{\mathbf{k}}) \mathbf{F}(\mathbf{r})=\varepsilon \mathbf{F}(\mathbf{r})$ (Slonczewski \& Weiss 1958, Ajiki \& Ando 1993, Ando 2005a), where $\mathbf{F}(\mathbf{r})$ is a two-component envelope function, $\gamma$ is a band parameter, $\hat{\mathbf{k}}=\left(\hat{k}_{x}, \hat{k}_{y}\right)=i \vec{\nabla}$ is a wave-vector operator, $\varepsilon$ is the energy, and $\sigma_{x}$ and $\sigma_{y}$ are the Pauli spin matrices. Two components of the wave function $\mathbf{F}(\mathbf{r})$ correspond to the amplitude at $A$ and $B$ sites in a unit cell. This $\mathbf{k} \cdot \mathbf{p}$ equation 

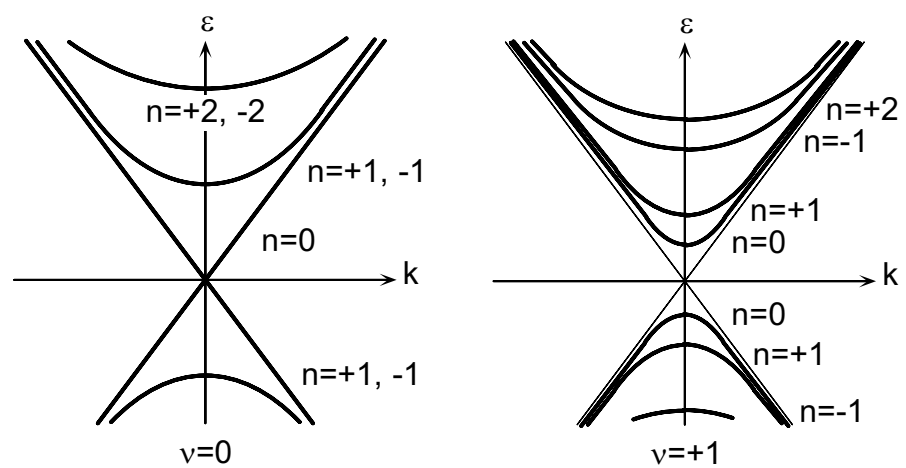

Figure 1. Energy bands of a nanotube obtained in the effective-mass approximation for metallic $\nu=0$ (left) and semiconducting $\nu=+1$ (right). In metallic nanotubes, the bands with $n= \pm 1, \pm 2, \cdots$ are doubly degenerate.

is the same as that for a massless neutrino or a relativistic Dirac electron with vanishing rest mass.

The electronic states of a nanotube can be obtained by imposing periodic boundary condition $\Psi(\mathbf{r}+\mathbf{L})=\Psi(\mathbf{r})$ except in extremely thin tubes, where $\mathbf{L}$ is the chiral vector determining the circumference. Under the translation $\mathbf{r} \rightarrow \mathbf{r}+\mathbf{L}$, the Bloch function at the $\mathrm{K}$ point changes its phase by $\exp (i \mathbf{K} \cdot \mathbf{L})=\exp (2 \pi i \nu / 3)$, where $\mathbf{K}$ is the wave vector at the $\mathrm{K}$ point and $\nu=0$ or \pm 1 depending on $\mathbf{L}$. Because $\Psi(\mathbf{r})$ is written as a product of the Bloch function and the neutrino wave function $\mathbf{F}(\mathbf{r})$, the boundary condition becomes $\mathbf{F}(\mathbf{r}+\mathbf{L})=\mathbf{F}(\mathbf{r}) \exp (-2 \pi i \nu / 3)$ so as to cancel the phase of the Bloch function. The phase can be ascribed to a fictitious AharonovBohm flux passing through the cross section. Therefore, an electron in a nanotube is regarded as a neutrino on the cylinder surface with a flux determined by the structure.

Energy levels in nanotubes for the K point are obtained by putting $k_{x}=\kappa_{\nu}(n)$ with $\kappa_{\nu}(n)=(2 \pi / L)[n-(\nu / 3)]$ and $k_{y}=k$ as $\varepsilon_{\nu}^{( \pm)}(n, k)= \pm \gamma \sqrt{\kappa_{\nu}(n)^{2}+k^{2}}$ (Ajiki \& Ando 1993), where $L=|\mathbf{L}|, n$ is an integer, and the upper (+) and lower ( -$)$ signs represent the conduction and valence bands, respectively. The Hamiltonian for the $\mathrm{K}^{\prime}$ point is obtained by replacing $\vec{\sigma}$ with $\vec{\sigma}^{*}$ and the boundary condition by replacing $\nu$ with $-\nu$ corresponding to $\mathbf{K}^{\prime}=-\mathbf{K}$, where $\mathbf{K}^{\prime}$ is the wave vector at the $K^{\prime}$ point. This shows that the nanotube becomes metallic for $\nu=0$ and semiconducting with gap $E_{g}=4 \pi \gamma / 3 L$ for $\nu= \pm 1$. Figure 1 shows a schematic illustration of the bands for $\nu=0$ and +1 in the vicinity of the $\mathrm{K}$ point.

An important feature is the presence of a topological singularity at $\mathbf{k}=0$. A neutrino has a helicity and its spin is quantized into the direction of the wave vector. The spin eigenfunction changes its sign under a $2 \pi$ rotation. Correspondingly, the pseudo-spin wave function acquires phase $-\pi$ due to Berry's phase when the wave vector $\mathbf{k}$ is rotated around the origin along a closed contour (Berry 1984, Ando et al 1998). The sign change occurs only when the contour encircles $\mathbf{k}=0$ but not when the contour does not contain $\mathbf{k}=0$. This topological singularity at $\mathbf{k}=0$ and Berry's phase are the origin of the absence of backscattering in metallic nanotubes as discussed below. 


\section{Absence of backward scattering}

Most scatterers including charged centers are characterized by a potential with range larger than the lattice constant $a=2.43 \AA$. For such scatterers, the same potential appears as the diagonal element of the matrix Hamiltonian and mixing between $\mathrm{K}$ and $\mathrm{K}^{\prime}$ points is safely neglected. In this case, the Born series for backward scattering vanish identically in graphene leading to the perfect conductance in metallic carbon nanotubes (Ando \& Nakanishi 1998). The perfect conductance has been confirmed by numerical calculations in a tight binding model (Nakanishi \& Ando 1999). In semiconducting nanotubes the resistance appears because the scattering does not correspond to the exact backscattering in graphene due to a nonzero Aharonov-Bohm flux.

Consider matrix elements of the Hamiltonian describing the potential of scatterers, given by a diagonal matrix in the pseudo-spin space. The direct matrix element between states with $\mathbf{k}$ and $\mathbf{- k}$ is proportional to the inner product of the pseudospin eigenfunctions with eigenvalues \pm 1 in the $\mathbf{k}$ direction and vanishes due to the orthogonality of the spin functions. In higher order each backscattering process corresponds to a $\pm \pi$ rotation $(\bmod 2 \pi)$ of the $\mathbf{k}$ direction. For each backscattering process, there always exists its time-reversal counterpart corresponding to the rotation in the opposite direction. The scattering amplitudes of these two processes are the same in the absolute value but have an opposite sign because of Berry's phase mentioned in the previous section. As a result, the backscattering amplitude cancels out completely even in higher order approximations.

The neutrino equation for $\mathbf{F}$ is invariant under the special time-reversal operation $S, \mathbf{F}^{S}=K \mathbf{F}^{*}$, where $\mathbf{F}^{*}$ stands for the complex conjugate of $\mathbf{F}$ and $K=-i \sigma_{y}$ satisfying $K^{2}=-1$. When $S$ is operated twice, the wave function changes its sign, i.e., $\mathbf{F}^{S^{2}}=\left(\mathbf{F}^{S}\right)^{S}=-\mathbf{F}$. The corresponding operation for operator $P$ is $P^{S}=K^{t} P K^{-1}$, where ${ }^{t} P$ stands for the transpose of $P$, i.e., ${ }^{t} P=\left(P^{*}\right)^{\dagger}$. We have $\left(\mathbf{F}_{\alpha}^{S}, P^{S} \mathbf{F}_{\beta}^{S}\right)=\left(\mathbf{F}_{\beta}, P \mathbf{F}_{\alpha}\right)$. This $S$ symmetry, characteristic to the symplectic universality class, is not destroyed by scatterers unless their potential range is not smaller than the lattice constant.

When the Fermi level moves away from the energy range where only linear bands are present, interband scattering appears because of the presence of several bands at the Fermi level. Let $r_{\bar{\beta} \alpha}$ be the reflection coefficient from a state with wave vector $\mathbf{k}_{\alpha}$ to a state with $\mathbf{k}_{\bar{\beta}} \equiv-\mathbf{k}_{\beta}$. Here, $\bar{\beta}$ stands for the state with wave vector opposite to $\beta$. We have $r_{\bar{\beta} \alpha}=\left(\mathbf{F}_{\beta}^{S}, T \mathbf{F}_{\alpha}\right)$ with $T=V+V G_{0} V+V G_{0} V G_{0} V+\cdots$, where $V$ is the impurity potential and $G_{0}$ is the Green's function in the absence of scatterers. The $T$ matrix satisfies $T^{S}=T$. With the use of the $S$ symmetry, we have $\left(\mathbf{F}_{\beta}^{S}, T \mathbf{F}_{\alpha}\right)=\left(\mathbf{F}_{\alpha}^{S}, T \mathbf{F}_{\beta}^{S^{2}}\right)=-\left(\mathbf{F}_{\alpha}^{S}, T \mathbf{F}_{\beta}\right)$, leading to the symmetry relation $r_{\bar{\beta} \alpha}=-r_{\bar{\alpha} \beta}$ (Ando \& Suzuura 2002).

Define the reflection matrix $r$ by $[r]_{\alpha \beta}=r_{\bar{\alpha} \beta}$. Then, we have $r=-{ }^{t} r$, where ${ }^{t} r$ is the transpose of $r$. In general we have $\operatorname{det}^{t} P=\operatorname{det} P$ for any matrix $P$, where $\operatorname{det} P$ is the determinant of $P$. In metallic nanotubes, the number of traveling modes is always given by an odd integer and therefore $\operatorname{det}(-r)=-\operatorname{det}(r)$, leading to $\operatorname{det}(r)=0$. By definition, $r_{\bar{\beta} \alpha}$ represents the amplitude of an out-going mode $\bar{\beta}$ with wave function $\psi_{\bar{\beta}}(\mathbf{r})$ for the reflected wave corresponding to an in-coming mode $\alpha$ with wave function $\psi_{\alpha}(\mathbf{r})$. Therefore, the vanishing determinant of $r$, i.e., the vanishing of an eigenvalue, shows the presence of a mode which is transmitted 
through the system without being scattered back. In fact, numerical calculations show that the conductance decreases with the length but remains larger than the ideal conductance $2 e^{2} / \pi \hbar$ for a single channel (Ando \& Suzuura 2002).

Inelastic scattering breaking the phase memory of an electron plays a very important role in destroying the perfect channel. In fact, the Boltzmann equation gives a finite conductivity when several bands coexist at the Fermi level although the conductivity becomes infinite in the energy range of the linear band (Ando \& Suzuura 2002). This is contrary to the presence of a perfect channel giving the ideal conductance $2 e^{2} / \pi \hbar$ for sufficiently long nanotubes. The difference originates from the absence of the phase coherence in the approach based on the transport equation. In fact, in the transport equation, scattering from each impurity is treated as a completely independent event after which an electron looses its phase memory. The perfect channel requires the phase coherence throughout the system. In the single-channel case, the transport equation gives an infinite conductivity because of the absence of the backscattering for each impurity.

The effects of inelastic scattering can be considered in a model in which the nanotube is separated into segments with length of the order of the phase coherence length $L_{\phi}$ and the electron looses the phase information after the transmission through each segment (Ando \& Suzuura 2002). The results show that as long as the length is smaller than or comparable to $L_{\phi}$, the conductance is close to the ideal value $2 e^{2} / \pi \hbar$ corresponding to the presence of a perfect channel. When the length becomes much larger than $L_{\phi}$, the conductance decreases in proportion to the inverse of the length and, therefore, an approximate conductivity proportional to $L_{\phi}$ can be defined.

\section{Absence of Drude tail}

The frequency dependence on the conductivity in the absence of backward scattering and in the presence of a perfect conducting channel is quite intriguing. The dynamical conductivity $\sigma(\omega)$ was calculated in a self-consistent Born approximation (SCBA) (Ando 2002) and a weak localization correction was also calculated (Suzuura \& Ando 2006). In SCBA, in particular, $\sigma(\omega)$ becomes a delta function, i.e., $\propto \delta(\omega)$, corresponding to the absence of backscattering, when the Fermi level lies in the linear bands in the weak scattering limit. When effects of other bands are included, however, $\sigma(\omega)$ deviates from $\delta(\omega)$ and starts to have a small Drude tail. When the Fermi level lies in higher bands, further, $\sigma(\omega)$ has a Drude form corresponding to finite relaxation time due to inter-band scattering and therefore fails to correctly describe the presence of a perfect channel.

One way to see the correct behavior of $\sigma(\omega)$ is an exact numerical diagonalization in systems with a finite size (Asada \& Ando 2006). We consider a metallic carbon nanotube with finite length $A$ (actually $A / L=100$ ), containing scatterers with a potential range larger than the lattice constant but smaller than the typical Fermi wavelength $(\sim 2 \pi / L)$. We impose periodic boundary conditions in the axis direction and restrict the basis by a cutoff energy $\epsilon_{\mathrm{c}}=3(2 \pi \gamma / L)$. The scattering strength is characterized by a dimensionless parameter $W$ proportional to the impurity concentration and the square of the potential strength of an individual impurity. 


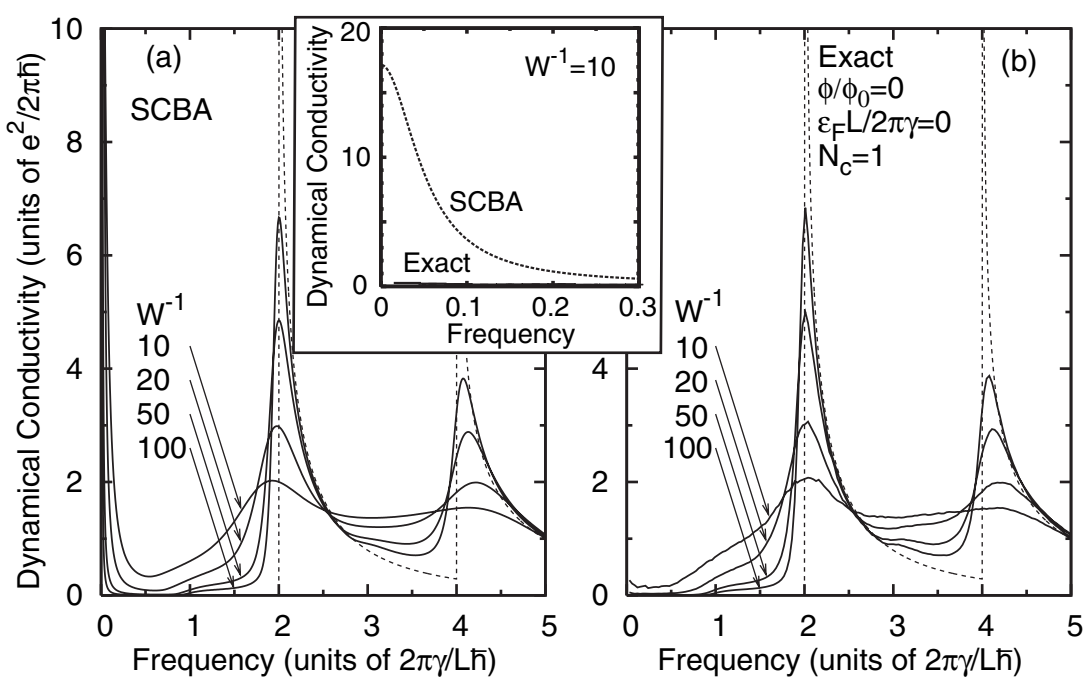

Figure 2. Dynamical conductivity for $\epsilon_{F} L / 2 \pi \gamma=0$ obtained in SCBA and by exact numerical diagonalization. The inset shows the blow-up in the low-frequency region.

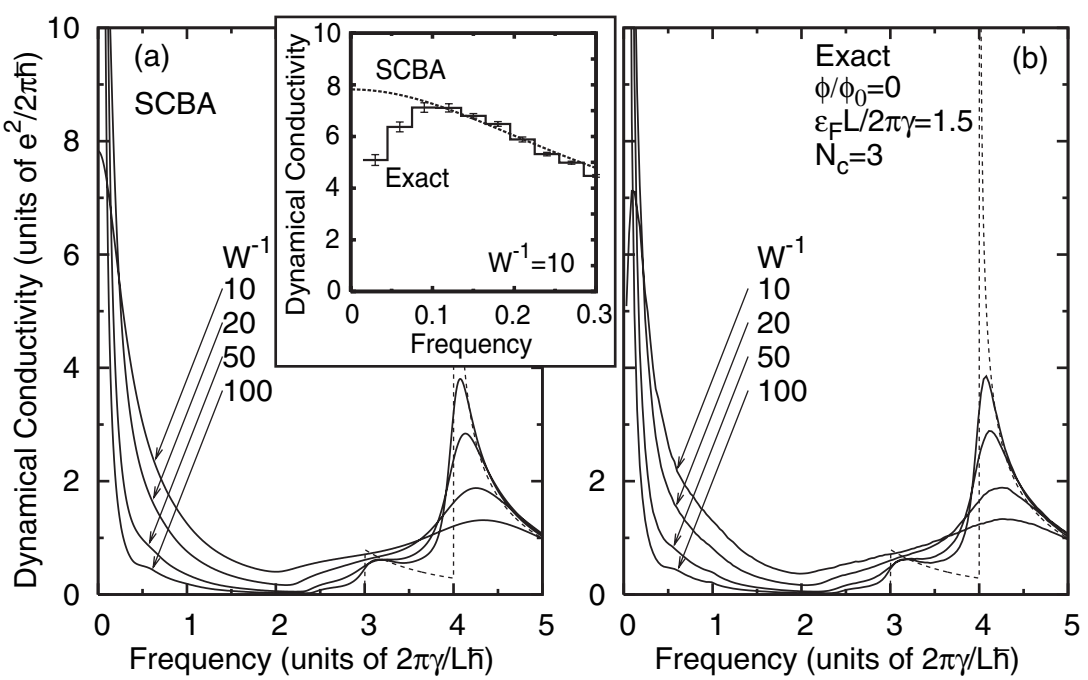

Figure 3. Dynamical conductivity for $\epsilon_{F} L / 2 \pi \gamma=1.5$ obtained in SCBA and by exact numerical diagonalization. The inset shows the blow-up in the low-frequency region.

Some examples of the results for $\varepsilon_{F}(2 \pi \gamma / L)^{-1}=0$ and 1.5 are shown in figures 2 and 3 , respectively. In the former case, there are only linear bands at the Fermi level corresponding to the absence of backscattering. In the latter case, there is a perfect conducting channel and the conductance of a finite-length nanotube decreases from the ideal value corresponding to the channel number three to that in the single channel case as the length of nanotube increases.

In figure 2, there is no significant difference between the results in SCBA and the exact results, when $\omega$ is comparable to or larger than $2 \pi \gamma / L \hbar$. The large van Hove 
structures corresponding to interband transitions present in regions $\hbar \omega(2 \pi \gamma / L)^{-1} \sim$ 2 and 4 are broadened due to impurities. There is a qualitative difference between the results in SCBA and the exact results near $\omega=0$. The Drude behavior with finite broadening appears in SCBA, while no such behavior is present in the exact calculation. The inset shows the expansion of the results near $\omega=0$. It is likely that the conductivity in the vicinity of $\omega=0$ is not broadened and there is a delta function at zero frequency, i.e., $\sigma(\omega)=\left(e^{2} \gamma / \hbar^{2} L\right) \delta(\omega)$ like the SCBA result in the weak scattering limit (Ando 2002).

Figure 3 shows results for $\varepsilon_{F}(2 \pi \gamma / L)^{-1}=1.5$. Except in the frequency region close to zero, the dynamical conductivity obtained by the exact diagonalization is nearly the same as that in SCBA, i.e., the Drude behavior is observed. On the other hand, in the small frequency region, the exact dynamical conductivity deviates from that in SCBA and decreases with the decrease of the frequency. Such a crossover occurs at $\omega_{\mathrm{c}} \approx 0.1 \times(2 \pi \gamma / L \hbar)$ in the case of $W^{-1}=10$. This corresponds to the crossover from the Drude transport regime to the "single-channel" transport regime. The result suggests again that there is a delta-function conductivity at zero frequency related to the perfect conducting channel. A detailed study on the presence of a delta function at zero frequency is highly desirable.

\section{Symmetry crossover}

The special time reversal $S$ is different from the real time reversal $T$ in which the $\mathrm{K}$ and $\mathrm{K}^{\prime}$ points are exchanged corresponding to the complex conjugate wave functions. In the present scheme, the $T$ operation gives $\mathbf{F}_{K}^{T}=e^{-i \psi} \sigma_{z} \mathbf{F}_{K^{\prime}}^{*}$ and $\mathbf{F}_{K^{\prime}}^{T}=$ $e^{-i \psi} \sigma_{z} \mathbf{F}_{K}^{*}$, where $\psi$ is an arbitrary phase, $\sigma_{z}$ is a Pauli spin matrix, and $\mathbf{F}_{K}$ and $\mathbf{F}_{K^{\prime}}$ are the neutrino wave functions for the K and K' points, respectively (Suzuura $\&$ Ando 2002, Ando 2006), When being repeated, the wave function returns to the original, i.e., $\left(\mathbf{F}_{K}^{T}\right)^{T}=\mathbf{F}_{K}$ and $\left(\mathbf{F}_{K^{\prime}}^{T}\right)^{T}=\mathbf{F}_{K^{\prime}}$. A system with this $T$ symmetry belongs to the orthogonal universality class.

In the presence of usual scatterers with their potential range larger than the lattice constant, the $\mathrm{K}$ and $\mathrm{K}$ ' points can be regarded as independent because their coupling can safely be neglected. In this case this $T$ symmetry is irrelevant and only the $S$ symmetry prevails. In the presence of short-range scatterers causing intervalley scattering between the $\mathrm{K}$ and $\mathrm{K}$ ' points, the $S$ symmetry is destroyed and the $T$ symmetry becomes relevant. Therefore, the symmetry crossover between the symplectic and orthogonal classes occurs due to short-range scatterers. The change from the anti-localization behavior in the symplectic case to the weak-localization behavior in the orthogonal case for the quantum correction to the conductivity has been demonstrated in graphene (Suzuura \& Ando 2002, McCann et al 2006).

The equi-energy line of graphene in the vicinity of the $\mathrm{K}$ or $\mathrm{K}$ ' points has a small trigonal warping. Effects of the warping can be included as a higher-order $\mathbf{k} \cdot \mathbf{p}$ term $\mathcal{H}_{1}$ proportional to $(k a)^{2}$ (Ajiki \& Ando 1996, Ando 2005). This Hamiltonian changes its sign under the $S$ operation, i.e., $\mathcal{H}_{1}^{S}=-\mathcal{H}_{1}$, and therefore destroys the $S$ symmetry. As a result, the symmetry of the system changes into unitary when the trigonal warping is appreciable. Further, the lattice strain and the nonzero curvature of the nanotube gives rise to an effective vector potential or Aharonov-Bohm flux, and therefore destroys the $S$ symmetry. 

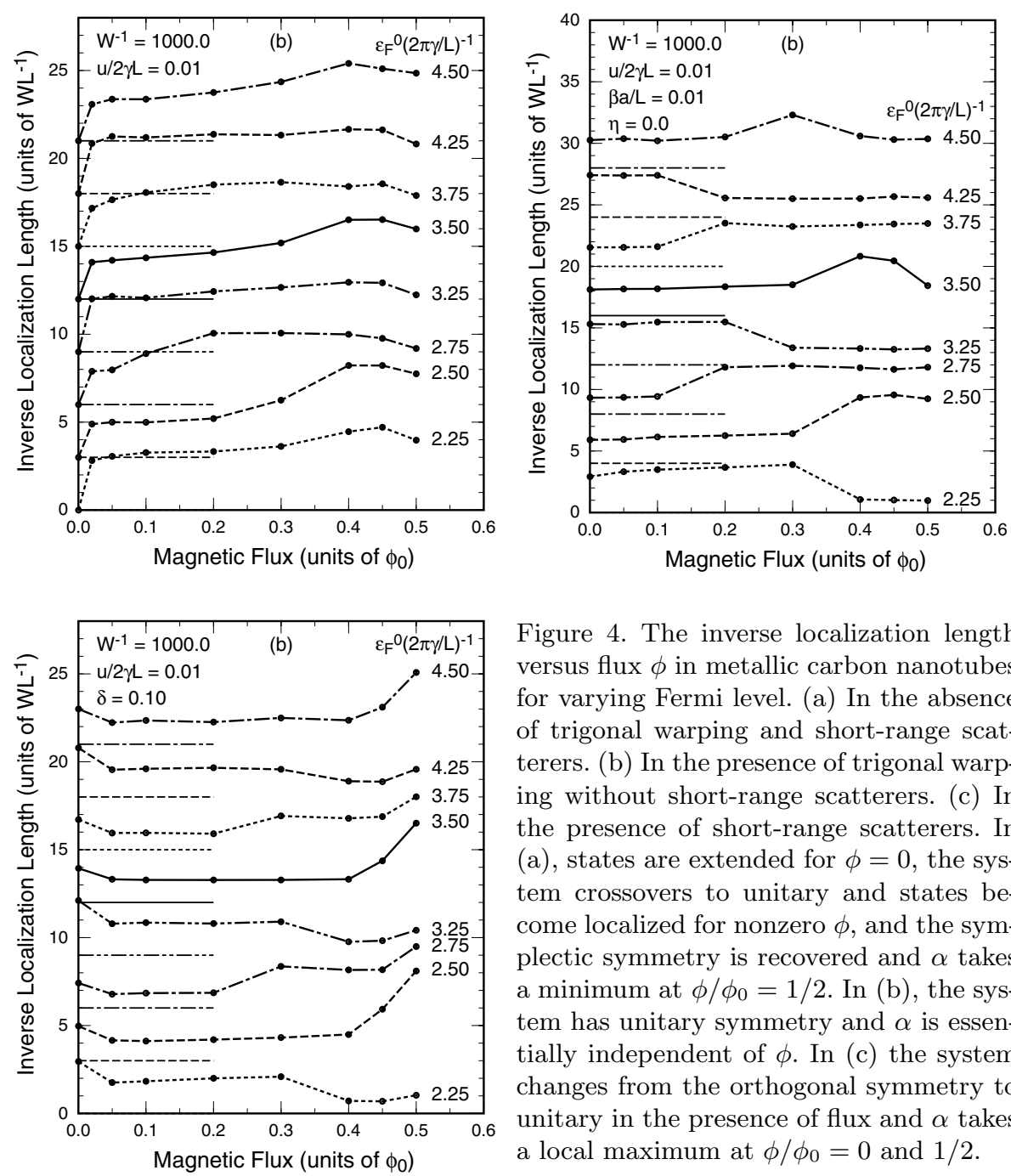

Figure 4. The inverse localization length versus flux $\phi$ in metallic carbon nanotubes for varying Fermi level. (a) In the absence of trigonal warping and short-range scatterers. (b) In the presence of trigonal warping without short-range scatterers. (c) In the presence of short-range scatterers. In (a), states are extended for $\phi=0$, the system crossovers to unitary and states become localized for nonzero $\phi$, and the symplectic symmetry is recovered and $\alpha$ takes a minimum at $\phi / \phi_{0}=1 / 2$. In (b), the system has unitary symmetry and $\alpha$ is essentially independent of $\phi$. In (c) the system changes from the orthogonal symmetry to unitary in the presence of flux and $\alpha$ takes a local maximum at $\phi / \phi_{0}=0$ and $1 / 2$.

This symmetry crossover of graphene manifests itself in the transport of a nanotube in the presence of Aharonov-Bohm flux (Ando 2006). In the presence of nonzero flux, the time reversal symmetry is destroyed and the symmetry changes into unitary except for $\phi / \phi_{0}=0$ and $\pi(\bmod 2 \pi)$ with flux quantum $\phi_{0}=c h / e$. This $\phi_{0} / 2$ oscillation was theoretically predicted to appear in the quantum correction of the conductivity (Altshuler et al 1981) and later observed experimentally in thin metal films on the surface of a cylinder (Sharvin \& Sharvin 1981). In metallic nanotubes, the states are exactly the same in the presence of an integer multiple of $\phi_{0}$, but not in the presence of $\phi_{0} / 2$ because of the change in the channel number. In fact, the channel number is odd, giving rise to a perfectly transmitting channel, in the absence of flux. In the presence of half-flux-quantum $\phi_{0} / 2$, on the other hand, the channel number becomes even and therefore no perfect channel can exist.

Figure 4 shows some examples of calculated inverse localization length $\alpha$ of 
a metallic nanotube as a function of the Aharonov-Bohm flux for various values of the electron concentration (Ando 2006). The electron concentration is specified by the Fermi energy $\varepsilon_{F}^{0}$ in the absence of flux. The inverse localization length is obtained by $\bar{G}(A) \propto \exp (-2 \alpha A)$ for sufficiently large $A$, where $\bar{G}$ is the geometric sample-average of the conductance and $A$ is the tube length.

In figure $4(\mathrm{a})$, there is neither trigonal warping nor short-range scatterer causing inter-valley scattering. For $\phi=0$, a perfect channel exists and therefore states are delocalized, giving $\alpha=0$. For nonzero $\phi$, the system crossovers to unitary from symplectic and therefore states become localized and $\alpha$ becomes nonzero. For $\phi / \phi_{0}=1 / 2$, the system recovers the symplectic symmetry and therefore $\alpha$ takes a minimum, which is nonzero because a perfect channel does not exist due to the even channel number.

Figure 4(b) shows the results in the presence of trigonal warping but without short-range scatterers. In this case the system has unitary symmetry even for $\phi / \phi_{0}=0$ and $1 / 2$. As a result the inverse localization length is essentially independent of the flux except for a small change due to the change in the channel number. Figure 4(c) shows the results in the presence of short-range scatterers. In this case the system changes from the orthogonal symmetry to unitary in the presence of flux. As a result the inverse localization length takes a local maximum at $\phi / \phi_{0}=0$ and $1 / 2$ corresponding to the orthogonal symmetry giving rise to stronger localization than the unitary symmetry.

\section{Summary}

In summary, an electron in a nanotube is a massless neutrino on a cylinder surface with a fictitious Aharonov-Bohm flux determined by the structure. A nanotube becomes a metal or a semiconductor, depending on whether the amount of the flux vanishes or not. One important feature of the neutrino equation is the presence of a topological singularity at the origin of the wave-vector space, leading to the absence of backward scattering and the presence of a perfectly conducting channel even in the presence of scatterers. Numerical calculations of the dynamical conductivity in finite-size systems reveal a singular dependence corresponding to the absence of backward scattering and the presence of a perfect channel. The absence of backscattering and the presence of a perfect channel can be understood in terms of the special time reversal symmetry. This symmetry can be destroyed by various effects such as short-range scatterers causing scattering between $\mathrm{K}$ and $\mathrm{K}$ ' point, trigonal warping, finite curvature, lattice distortion, etc. The symmetry crossover has been demonstrated by the Aharonov-Bohm effect on the localization effect in metallic nanotubes.

This work was supported in part by a 21st Century COE Program at Tokyo Tech "Nanometer-Scale Quantum Physics" and by Grant-in-Aid for Scientific Research from Ministry of Education, Culture, Sports, Science and Technology, Japan.

\section{References}

Ajiki, H. \& Ando, T. 1993 J. Phys. Soc. Jpn. 62, 1255.

Ajiki, H. \& Ando, T. 1996 J. Phys. Soc. Jpn 65, 505.

Altshuler, B. L., Aronov, A. G. \& Spivak, B. Z. 1981 JETP Lett. 33, 94.

Article submitted to Royal Society 
Ando, T. 2002 J. Phys. Soc. Jpn. 71, 2505.

Ando, T. 2005 J. Phys. Soc. Jpn. 74, 777.

Ando, T. 2006 J. Phys. Soc. Jpn. 75, 054701.

Ando, T. \& Nakanishi, T. 1998 J. Phys. Soc. Jpn. 67, 1704.

Ando, T. \& Suzuura, H. 2002 J. Phys. Soc. Jpn. 71, 2753.

Ando, T., Nakanishi, T. \& Saito, R. 1998 J. Phys. Soc. Jpn. 67, 2857.

Asada, Y. \& Ando, T. 2006 J. Phys. Soc. Jpn. 75, 094711.

Berry, M. V. 1984 Proc. Roy. Soc. London A392, 45.

McCann, E., Kechedzhi, K., Falko, V. I., Suzuura, H., Ando, T. \& Altshuler, B. L. 2006 Phys. Rev. Lett. 97, 146805.

Nakanishi, T. \& Ando, T. 1999 J. Phys. Soc. Jpn. 68, 561.

Sharvin, D. Yu. \& Sharvin, Yu. V. 1981 JETP Lett. 34, 272.

Slonczewski, J. C. \& Weiss, P. R. 1958 Phys. Rev. 109, 272.

Suzuura, H. \& Ando, T. 2002 Phys. Rev. Lett. 89, 266603.

Suzuura, H. \& Ando, T. 2006 J. Phys. Soc. Jpn. 75, 024703.

Wallace, P. R. 1947 Phys. Rev. 71, 622. 